\title{
The Perron-Frobenius operators, invariant measures and representations of the Cuntz-Krieger algebras
}

\author{
KATSUNORI KAWAMURA \\ Research Institute for Mathematical Sciences \\ Kyoto University, Kyoto 606-8502, Japan
}

For a transformation $F$ on a measure space $(X, \mu)$, we show that the Perron-Frobenius operator of $F$ can be written by a representation $\left(L_{2}(X, \mu), \pi\right)$ of the Cuntz-Krieger algebra $\mathcal{O}_{A}$ associated with $F$ when $F$ satisfies some assumption. Especially, when $\mathcal{O}_{A}$ is the Cuntz algebra $\mathcal{O}_{N}$ and $\left(L_{2}(X, \mu), \pi\right)$ in the above is some irreducible representation of $\mathcal{O}_{N}$, then there is an $\boldsymbol{F}$-invariant measure on $\boldsymbol{X}$ which is absolutely continuous with respect to $\mu$.

\section{Introduction}

Invariant measures (especially, Haar measures) play an important role in the representation theory of Lie groups and harmonic analysis. On the other hand, invariant measures of non invertible transformations are studied in $[\mathbf{4}, \mathbf{5}, \mathbf{6}]$ by the Perron-Frobenius operators of dynamical systems. By using the Perron-Frobenius operators, the characterization of a given dynamical system and the construction of invariant measure are obtained. We show their roles in representation theory of operator algebras in this paper.

Let $L_{p}(X, \mu)$ be the set of all complex-valued measurable functions $\phi$ on a measure space $(X, \mu)$ satisfying $\|\phi\|_{L_{p}}<\infty$ and let $L_{p}(X, \mu ; \mathbf{R})$ be the subset of all real-valued functions in $L_{p}(X, \mu)$ for $p=1,2, \infty$. For a nonsingular transformation $F$ on $X$ (that is, $\mu\left(F^{-1}(A)\right)=0$ if $\mu(A)=0$ for $A \subset X), P_{F}$ is the Perron-Frobenius operator (or the Frobenius-Perron operator, the transfer operator) of $F$ if $P_{F}$ is the operator on $L_{1}(X, \mu)$ which satisfies

$$
\int_{A}\left(P_{F} \psi\right)(x) d \mu(x)=\int_{F^{-1}(A)} \psi(x) d \mu(x) \quad\left(\forall \psi \in L_{1}(X, \mu)\right)
$$

for each measurable subset $A$ of $X([5])$. By (1.1), $P_{F} \psi$ is uniquely determined as an element in $L_{1}(X, \mu)$ for each $\psi \in L_{1}(X, \mu)$. For $\psi \in L_{1}(X, \mu)$ and $\theta \in L_{\infty}(X, \mu)$, we obtain $\int_{X} \theta(F(x)) \psi(x) d \mu(x)=\int_{X} \theta(x)\left(P_{F} \psi\right)(x) d \mu(x)$. ¿From this, $P_{F}$ is a bounded linear operator on $L_{1}(X, \mu)$ and $\left\|P_{F} \psi\right\|_{L_{1}} \leq$

e-mail:kawamura@kurims.kyoto-u.ac.jp. 
$\|\psi\|_{L_{1}}$ for each $\psi \in L_{1}(X, \mu ; \mathbf{R})$. Further, a positive function $\rho \in L_{1}(X, \mu)$ satisfies $P_{F} \rho=\rho$ if and only if $\rho$ is the density of an $F$-invariant measure, that is, the following holds for any $\psi \in L_{1}(X, \mu)$ :

$$
\int_{X} \psi(F(x)) \rho(x) d \mu(x)=\int_{X} \psi(x) \rho(x) d \mu(x) .
$$

In order to describe both the Perron-Frobenius operators and representations of the Cuntz-Krieger algebras simultaneously, we introduce branching function systems on a measure space $(X, \mu)$. A family $f=\left\{f_{i}\right\}_{i=1}^{N}$ of maps on $X$ is a semibranching function system if there is a finite family $\left\{D_{i}\right\}_{i=1}^{N}$ of measurable subsets of $X$ such that $f_{i}$ is a measurable map from $D_{i}$ to $R_{i} \equiv f_{i}\left(D_{i}\right), \mu\left(X \backslash R_{1} \cup \cdots \cup R_{N}\right)=0, \mu\left(R_{i} \cap R_{j}\right)=0$ when $i \neq j$ and there is the Radon-Nikodým derivative $\Phi_{f_{i}}$ of $\mu \circ f_{i}$ with respect to $\mu$ and $\Phi_{f_{i}}>0$ almost everywhere in $D_{i}$ for $i=1, \ldots, N$. A map $F$ on $X$ is called the coding map of a semibranching function system $f=\left\{f_{i}\right\}_{i=1}^{N}$ if $F \circ f_{i}=i d_{D_{i}}$ for $i=1, \ldots, N$.

For a semibranching function system $f=\left\{f_{i}\right\}_{i=1}^{N}$ with the coding map $F$, define a family $\left\{S\left(f_{i}\right)\right\}_{i=1}^{N}$ of operators on $L_{2}(X, \mu)$ by

$$
\left(S\left(f_{i}\right) \phi\right)(x) \equiv \chi_{R_{i}}(x) \cdot\left\{\Phi_{F}(x)\right\}^{1 / 2} \cdot \phi(F(x)) \quad\left(\phi \in L_{2}(X, \mu)\right)
$$

where $\chi_{R_{i}}$ is the characteristic function of $R_{i}$. Then $S\left(f_{i}\right)$ is a partial isometry with the initial space $L_{2}\left(D_{i}, \mu\right)$ and the final space $L_{2}\left(R_{i}, \mu\right)$, and $S\left(f_{i}\right) S\left(f_{j}\right)=S\left(f_{i} \circ f_{j}\right)$ when $D_{j} \subset R_{i}$. For $N \geq 2$, let $A$ be an $N \times N$ matrix which consists of elements 0 or 1 and any column and row are not 0 . A semibranching function system $f=\left\{f_{i}\right\}_{i=1}^{N}$ is an A-branching function system if $\mu\left(D_{i} \backslash \bigcup_{j ; a_{i j}=1} R_{j}\right)=0$ for each $i=1, \ldots, N$. For an $A$-branching function system $f=\left\{f_{i}\right\}_{i=1}^{N}$,

$$
\pi_{f}\left(s_{i}\right) \equiv S\left(f_{i}\right) \quad(i=1, \ldots, N),
$$

defines a representation $\left(L_{2}(X, \mu), \pi_{f}\right)$ of the Cuntz-Krieger algebra $\mathcal{O}_{A}$.

Theorem 1.1. For an A-branching function system $f=\left\{f_{i}\right\}_{i=1}^{N}$ with the coding map $F$, the following holds:

$$
\left(P_{F} \psi\right)(x)=\left\{\left(\pi_{f}\left(s_{1}^{*}\right) \sqrt{\psi}\right)(x)\right\}^{2}+\cdots+\left\{\left(\pi_{f}\left(s_{N}^{*}\right) \sqrt{\psi}\right)(x)\right\}^{2}
$$

for any positive function $\psi \in L_{1}(X, \mu)$ where $\sqrt{\psi}(x) \equiv \sqrt{\psi(x)}$.

Theorem 1.2. Assume that $F$ is the coding map of an A-branching function system $f=\left\{f_{i}\right\}_{i=1}^{N}$ on a measure space $(X, \mu)$ and $b_{i} \equiv \Phi_{f_{i}}$ is constant for $i=1, \ldots, N$ and $\mu(X)<\infty$. Then the following holds:

(i) Define a subspace $V \equiv \operatorname{Lin}<\left\{\chi_{R_{1}}, \ldots, \chi_{R_{N}}\right\}>$ of $L_{2}(X, \mu)$. Then $P_{F} V \subset V$ where $R_{i}$ is the image of $f_{i}$. 
(ii) For a diagonal matrix $B \equiv \operatorname{diag}\left(b_{1}, \ldots, b_{N}\right) \in M_{N}(\mathbf{R})$, the following identity of matrices holds:

$$
\left.P_{F}\right|_{V}=B A
$$

where $\left.P_{F}\right|_{V}$ is the matrix representation over the basis $\chi_{R_{1}}, \ldots, \chi_{R_{N}}$ of $V$ and the rhs is the product of matrices.

In Theorem 1.2 (ii), the eigenvalues of the Perron-Frobenius operator associated with $F$ depend on not only $A$ but also $B$. In this sense, eigenvalues of the Perron-Frobenius operator have the information of a representation of the Cuntz-Krieger algebra.

It is important problem to construct the invariant measure for a given dynamical system. For example, Lasota-York theorem shows a construction of invariant measure by using the Perron-Frobenius operator of a dynamical system $([4])$. We show the condition of existence of invariant measure from the viewpoint of representation theory of the Cuntz algebra.

$f=\left\{f_{i}\right\}_{i=1}^{N}$ is a branching function system if $f=\left\{f_{i}\right\}_{i=1}^{N}$ is an $A$ branching function system for a matrix $A=\left(a_{i j}\right), a_{i j}=1$ for each $i, j=$ $1, \ldots, N$. In this case, $\left(L_{2}(X, \mu), \pi_{f}\right)$ is a representation of the Cuntz algebra $\mathcal{O}_{N}$. For $z=\left(z_{i}\right)_{i=1}^{N} \in S^{N-1} \equiv\left\{y \in \mathbf{R}^{N}:\|y\|=1\right\},(\mathcal{H}, \pi)$ is $G P(z)$ of $\mathcal{O}_{N}$ if there is a unit cyclic vector $\Omega \in \mathcal{H}$ such that

$$
\pi\left(z_{1} s_{1}+\cdots+z_{N} s_{N}\right) \Omega=\Omega .
$$

We call $\Omega$ by the $G P$ vector of $(\mathcal{H}, \pi)$. In this case, there is $g \in O(N) \subset U(N)$ such that $\left(\pi \circ \alpha_{g}\right)\left(s_{1}\right) \Omega=\Omega$ where $\alpha$ is the canonical action of $U(N)$ on $\mathcal{O}_{N}$. This implies that $\left(\mathcal{H}, \pi \circ \alpha_{g}\right)$ is an irreducible permutative representation of $\mathcal{O}_{N}([\mathbf{1}])$. Hence $G P(z)$ of $\mathcal{O}_{N}$ exists uniquely up to unitary equivalence and it is irreducible. Further we see that $G P(z) \sim G P(y)$ if and only if $z=y$ where $\sim$ means the unitary equivalence.

Theorem 1.3. Let $F$ be the coding map of a branching function system $f$ on a measure space $(X, \mu)$. If there is $z \in S^{N-1}$ such that $\left(L_{2}(X, \mu), \pi_{f}\right)$ is $G P(z)$ with the GP vector $\Omega \in L_{2}(X, \mu ; \mathbf{R})$, then there is a probabilistic $F$-invariant measure $\nu$ on $X$ which is absolutely continuous with respect to $\mu$ and it is given as follows:

$$
d \nu(x) \equiv\{\Omega(x)\}^{2} d \mu(x) \quad(x \in X) .
$$

In $\S 2$, we show the main theorems. It is explained that (1.5) implies the eigenequation of the Perron-Frobenius operator. In $\S 3$, we show concrete examples.

\section{Proofs of the main theorems}

For $N \geq 2$, let $M_{N}(\{0,1\})$ be the set of all $N \times N$ matrices such that each element is 0 or 1 and any row and column is not 0 . For $A=\left(a_{i j}\right) \in M_{N}(\{0,1\})$, 
$\mathcal{O}_{A}$ is the Cuntz-Krieger algebra by $A$ if $\mathcal{O}_{A}$ is a $\mathrm{C}^{*}$-algebra which is universally generated by generators $s_{1}, \ldots, s_{N}$ and they satisfy $s_{i}^{*} s_{i}=\sum_{j=1}^{N} a_{i j} s_{j} s_{j}^{*}$ for $i=1, \ldots, N$ and $\sum_{i=1}^{N} s_{i} s_{i}^{*}=I([\mathbf{3}])$. Especially, when $a_{i j}=1$ for each $i, j=1, \ldots, N, \mathcal{O}_{A}$ is the Cuntz algebra $\mathcal{O}_{N}([\mathbf{2}])$. In this paper, any representation is unital and $*$-preserving.

Proof of Theorem 1.1. By (1.3), the adjoint operator $S\left(f_{i}\right)^{*}$ of $S\left(f_{i}\right)$ on $L_{2}(X, \mu)$ is as follows:

$$
\left(S\left(f_{i}\right)^{*} \phi\right)(x)=\chi_{D_{i}}(x) \cdot\left\{\Phi_{f_{i}}(x)\right\}^{1 / 2} \cdot \phi\left(f_{i}(x)\right) \quad\left(\phi \in L_{2}(X, \mu)\right) .
$$

For the coding map $F$ of a semibranching function system $f=\left\{f_{i}\right\}_{i=1}^{N}$, we have

$$
\left(P_{F} \psi\right)(x)=\sum_{i=1}^{N} \chi_{D_{i}}(x) \cdot \Phi_{f_{i}}(x) \cdot \psi\left(f_{i}(x)\right) \quad\left(\psi \in L_{1}(X, \mu)\right) .
$$

By (2.1) and (2.2), we have

$$
\left(P_{F} \psi\right)(x)=\left\{\left(S\left(f_{1}\right)^{*} \sqrt{\psi}\right)(x)\right\}^{2}+\cdots+\left\{\left(S\left(f_{N}\right)^{*} \sqrt{\psi}\right)(x)\right\}^{2}
$$

for any positive function $\psi \in L_{1}(X, \mu)$. By (1.4) and (2.3), the statement holds.

Proof of Theorem 1.2. Define $v_{i} \equiv \chi_{R_{i}}$ for $i=1, \ldots, N$.

(i) We see that $\chi_{D_{i}}=\sum_{k=1}^{N} a_{i k} v_{k}$. By $(2.1),\left(S\left(f_{i}\right)^{*} \phi\right)(x)=\sqrt{b_{i}} \chi_{D_{i}}(x) \phi\left(f_{i}(x)\right)$. ¿From this, $S\left(f_{i}\right)^{*} v_{j}=\sum_{k=1}^{N} b_{i}^{-1 / 2} c_{i k}^{(j)} v_{k}$ for $i=1, \ldots, N$ where $c_{i k}^{(j)}=$ $\delta_{i j} b_{i} a_{i k}$. Hence $S\left(f_{i}\right)^{*} V \subset V$. By (2.2), the following holds:

$$
P_{F}=b_{1}^{1 / 2} S\left(f_{1}\right)^{*}+\cdots+b_{N}^{1 / 2} S\left(f_{N}\right)^{*} .
$$

Therefore the statement is proved.

(ii) By the proof of (i), we see that $\left.S\left(f_{i}\right)^{*}\right|_{V}=\left(b_{i}^{-1 / 2} c_{j k}^{(i)}\right)$ as a matrix with respect to $v_{1}, \ldots, v_{N}$. From $(2.4)$, the statement holds.

Corollary 2.1. Let $X$ be a bounded closed interval of $\mathbf{R}$ and $A \in M_{N}(\{0,1\})$. Assume that $f=\left\{f_{i}\right\}_{i=1}^{N}$ is an A-branching function system on $X$ and $b_{i} \equiv \Phi_{f_{i}}$ is constant for each $i=1, \ldots, N$. Then the eigenvalue of $B A$ becomes that of the Perron-Frobenius operator of the coding map $F$ of $f$ where $B \equiv \operatorname{diag}\left(b_{1}, \ldots, b_{N}\right)$.

Proof of Theorem 1.3. Assume that $\Omega \in L_{2}(X, \mu ; \mathbf{R})$ satisfies $\pi_{f}\left(z_{1} s_{1}+\right.$ $\left.\cdots+z_{N} s_{N}\right) \Omega=\Omega$. Define $\rho(x) \equiv(\Omega(x))^{2}$ for $x \in X$. Then $\rho \in L_{1}(X, \mu)$. By Theorem 1.1 and $\pi_{f}\left(s_{i}\right)^{*} \Omega=\pi_{f}\left(s_{i}\right)^{*} \pi_{f}\left(z_{1} s_{1}+\cdots+z_{N} s_{N}\right) \Omega=z_{i} \Omega$, we have $\left(P_{F} \rho\right)(x)=\sum_{i=1}^{N}\left\{\left(\pi\left(s_{i}\right)^{*} \Omega\right)(x)\right\}^{2}=\sum_{i=1}^{N}\left\{z_{i} \Omega(x)\right\}^{2}=\rho(x)$. Hence 
$P_{F} \rho=\rho$. This implies the statement.

Corollary 2.2. Let $X$ be a measurable subset of $\mathbf{R}$. Assume that a piecewise $C^{1}$-class map $F$ on $X$ is the coding map of a branching function system $\left\{f_{i}\right\}_{i=1}^{N}$ on the measure space $(X, d x)$ where $d x$ is the Lebesgue measure. If $\phi_{0} \in L_{2}(X, d x ; \mathbf{R})$ satisfies

$$
\sqrt{\left|F^{\prime}(x)\right|} \phi_{0}(F(x))=\sqrt{N} \phi_{0}(x) \quad(\text { a.e. } x \in X),
$$

then $d \mu(x) \equiv\left\{\phi_{0}(x)\right\}^{2} d x$ is an invariant measure on $X$ with respect to $F$.

Proof. By (1.3) and (1.4), we see that $\left(\pi_{f}\left(s_{1}+\cdots+s_{N}\right) \phi\right)(x)=$

$\sqrt{\left|F^{\prime}(x)\right|} \phi(F(x))$ for each $\phi \in L_{2}(X, d x)$. ¿From this and $(2.5), \pi_{f}\left(N^{-1 / 2} s_{1}+\right.$ $\left.\cdots+N^{-1 / 2} s_{N}\right) \phi_{0}=\phi_{0}$. By Theorem 1.3 for $z=\left(N^{-1 / 2}, \ldots, N^{-1 / 2}\right) \in$ $S^{N-1}$, the statement holds.

In $\S 6.5$ of [5], it is explained that intertwiners among dynamical systems bring new invariant measures from known ones. We show its unitary version as follows:

Proposition 2.3. Let $F$ be the coding map of a branching function system $f=\left\{f_{i}\right\}_{i=1}^{N}$ on a measure space $(X, \mu)$. Assume that $\left(L_{2}(X, \mu), \pi_{f}\right)$ is $G P(z)$ for $z \in S^{N-1}$ with the GP vector $\Omega \in L_{2}(X, \mu ; \mathbf{R})$. If $\zeta$ is a measure space isomorphism from $(X, \mu)$ to other $(Y, \nu)$ and $G \equiv \zeta \circ F \circ \zeta^{-1}$, then $\rho \equiv$ $(S(\zeta) \Omega)^{2}$ is the density of a probabilistic $G$-invariant measure on $Y$ which is absolutely continuous with respect to $\nu$ where $S(\zeta)$ is a unitary operator from $L_{2}(X, \mu)$ to $L_{2}(Y, \nu)$ defined by $(S(\zeta) \phi)(y) \equiv\left\{\Phi_{\zeta^{-1}}(y)\right\}^{1 / 2} \phi\left(\zeta^{-1}(y)\right)$.

Proof. Define a branching function system $g=\left\{g_{i}\right\}_{i=1}^{N}$ by $g_{i} \equiv \zeta \circ f_{i} \circ$ $\zeta^{-1}$. Then $G$ is the coding map of $g$. We see that $S(\zeta) \pi_{f}(\cdot) S(\zeta)^{*}=\pi_{g}(\cdot)$, $\pi_{g}\left(z_{1} s_{1}+\cdots+z_{N} s_{N}\right) \Omega^{\prime}=\Omega^{\prime}$ for $\Omega^{\prime} \equiv S(\zeta) \Omega \in L_{2}(Y, \nu ; \mathbf{R})$. Hence $\left(L_{2}(Y, \nu), \pi_{g}\right)$ is $G P(z)$ with the GP vector $\Omega^{\prime}$. By Theorem 1.3, we have the statement.

\section{Examples}

Example 3.1. Let $0<a<1$ and $X \equiv[0,1]$.

(i) Define a map $F$ on $X$ by $F(x) \equiv x / a$ on $R_{1} \equiv[0, a]$ and $F(x) \equiv$ $-(x-1) /(1-a)$ on $R_{2} \equiv[a, 1]$. 

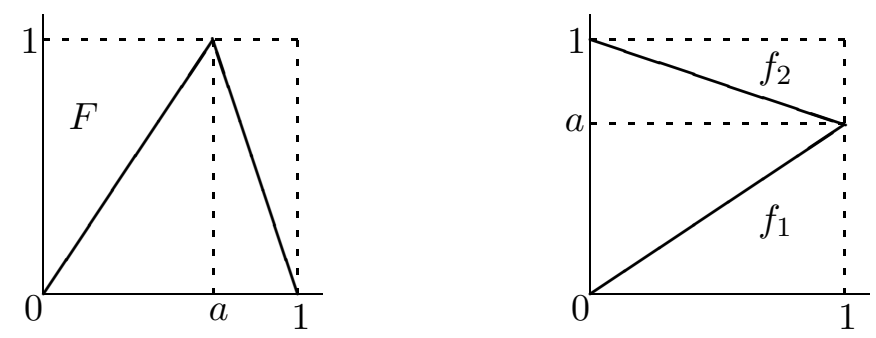

Then $F$ is the coding map of a branching function system $f \equiv\left\{f_{1}, f_{2}\right\}$ defined by $f_{i} \equiv\left(\left.F\right|_{R_{i}}\right)^{-1}$ for $i=1,2$. Then $\left(\pi_{f}\left(s_{1}\right) \phi\right)(x)=a^{-1 / 2} \chi_{R_{1}}(x) \phi(x / a)$, $\left(\pi_{f}\left(s_{2}\right) \phi\right)(x)=(1-a)^{-1 / 2} \chi_{R_{2}}(x) \phi(-(x-1) /(1-a))$ for $\phi \in L_{2}(X, d x)$.

$\left(P_{F} \psi\right)(x)=a \psi(a x)+(1-a) \psi(-(1-a) x+1) \quad\left(\psi \in L_{1}(X, d x)\right)$.

The Lebesgue measure $d x$ is the probabilistic invariant measure of $X$ with respect to $F$.

(ii) Define $f_{1}(x) \equiv \sqrt{a} x$ and $f_{2}(x) \equiv \sqrt{(1-a) x^{2}+a}$ on $X$. The coding map $F$ of $f=\left\{f_{1}, f_{2}\right\}$ is given by $F(x)=x / \sqrt{a}$ on $[0, \sqrt{a}]$, $F(x)=\sqrt{\left(x^{2}-a\right) /(1-a)}$ on $[\sqrt{a}, 1]$. Then a function $\Omega(x) \equiv \sqrt{2 x}$ on $[0,1]$ satisfies $\pi_{f}\left(\sqrt{a} s_{1}+\sqrt{1-a} s_{2}\right) \Omega=\Omega$. Hence the probabilistic $F$-invariant measure on $X$ is $2 x d x$.

$\left(L_{2}(X, d x), \pi_{f}\right)$ in both (i) and (ii) is $G P(\sqrt{a}, \sqrt{1-a})$ of $\mathcal{O}_{2}$. Both invariant measures are independent in the parameter $a$.

Example 3.2. For $a, b \in \mathbf{R}, a \neq 0$, define

$$
F(x) \equiv(x-b)^{2} / a+b-2 a
$$

on $X \equiv[-2|a|+b, 2|a|+b]$. Define a branching function system $f=\left\{f_{1}, f_{2}\right\}$ on $X$ by $f_{i} \equiv\left(\left.F\right|_{R_{i}}\right)^{-1}, i=1,2$ for $R_{1} \equiv[-2|a|+b, b]$ and $R_{2} \equiv[b, 2|a|+b]$. Then $\pi_{f}\left(s_{1}+s_{2}\right) \Omega=\Omega$ for $\Omega(x) \equiv \pi^{-1 / 2}\left\{4 a^{2}-(x-b)^{2}\right\}^{-1 / 4}$. Hence

$$
\rho(x) \equiv \frac{1}{\pi} \frac{1}{\sqrt{4 a^{2}-(x-b)^{2}}}
$$

is the density of a probabilistic invariant measure on $X$ with respect to $F$. When $a=-1 / 4$ and $b=1 / 2$, we have

$$
F(x)=4 x(1-x), \quad \rho(x)=\frac{1}{\pi} \frac{1}{\sqrt{x(1-x)}} .
$$

This was first obtained by Ulman and von Neumann ([8]). 

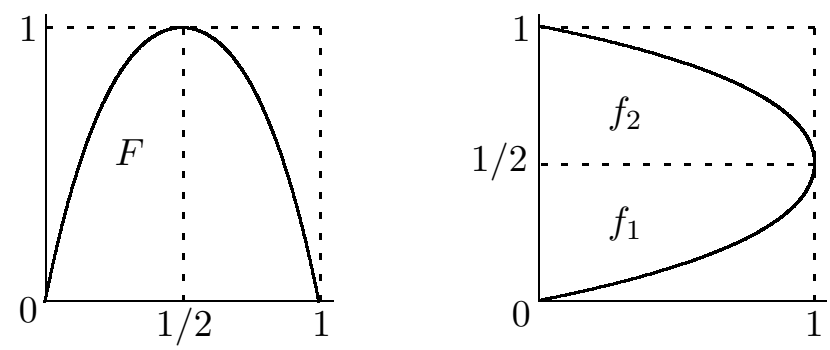

Example 3.3. For $0<a<1$, define a map $F:[0,1] \rightarrow[0,1]$ as follows:

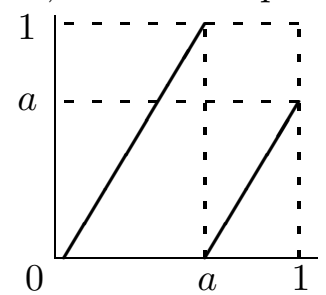

Define $R_{1} \equiv[0, a], R_{2} \equiv[a, 1], D_{1} \equiv[0,1], D_{2} \equiv[0, a]$. Then $F$ is the coding map of the following $A=\left(\begin{array}{ll}1 & 1 \\ 1 & 0\end{array}\right)$-branching function system on $X=[0,1]: f_{i}: D_{i} \rightarrow R_{i}, f_{1}(x)=a x$ for $x \in[0,1]$ and $f_{2}(x)=(1-a) x / a+a$ for $x \in[0, a]$. Define $v_{1} \equiv \chi_{[0, a]}, v_{2} \equiv \chi_{[a, 1]}, V \equiv \operatorname{Lin}<\left\{v_{1}, v_{2}\right\}>$. Then the matrix representation of $P_{F}$ with respect to $v_{1}, v_{2}$ is given as follows:

$$
\left.P_{F}\right|_{V}=\left(\begin{array}{cc}
a & (1-a) / a \\
a & 0
\end{array}\right)
$$

Hence its eigenvalue are $a-1$ and 1 . Their normalized eigenvectors are given as follows:

$w_{1}=\sqrt{\frac{1-a}{a}} \chi_{[0, a]}-\sqrt{\frac{a}{1-a}} \chi_{[a, 1]}, \quad w_{2}=\frac{1}{\sqrt{a\left(1+a-a^{2}\right)}}\left(\chi_{[0, a]}+a \chi_{[a, 1]}\right)$.

Especially $w_{2}$ is the density of the invariant measure on $[0,1]$ with respect to $F$.

Example 3.4. We show applications of Proposition 2.3. The following $(X, F, \mu)$ is a transformation $F$ on $X \subset \mathbf{R}$ and a probabilistic invariant measure $\mu$ on $X$ :

(i) For $b \in \mathbf{R} \backslash[-1,0], X \equiv[0,1]$,

$$
F(x) \equiv \begin{cases}2 b^{2} /(b-x)-2 b & \left(x \in D_{1}\right), \\ \frac{2 b^{2}}{1+3 b}\left\{\frac{(1+b)^{2}}{(1+3 b) x+b(b-1)}-1\right\} & \left(x \in D_{2}\right),\end{cases}
$$


where $D_{1} \equiv[0, b /(2 b+1))$ and $D_{2} \equiv[b /(2 b+1), 1]$. Then

$$
d \mu(x)=\frac{b(b+1)}{(x+b)^{2}} d x \quad(x \in[0,1]) .
$$

(ii) For $0 \leq k<1, X \equiv[-1,1]$,

$$
F(x) \equiv \frac{2 x^{2}}{1-k^{2}\left(1-x^{2}\right)^{2}}-1 . \quad \text { Then } d \mu(x)=\frac{1}{2 K} \frac{d x}{\sqrt{\left(1-x^{2}\right)\left(1-k^{2}\left(1-x^{2}\right)\right)}}
$$

where $K$ is the positive constant defined by

$$
K \equiv \int_{0}^{1} \frac{1}{\sqrt{\left(1-x^{2}\right)\left(1-k^{2} x^{2}\right)}} d x .
$$

(iii) For an integer $N \geq 2, X \equiv[0,1]$ and

$$
F(x) \equiv(N \sqrt{x}-[N \sqrt{x}])^{2}, \text { we have } d \mu(x)=\frac{d x}{2 \sqrt{x}}
$$

where [.] is the greatest integer less than equal $x$.

(iv) For a real number $a>1, X \equiv[1, a]$ and

$$
F(x) \equiv a / x^{2} \quad(x \in[1, \sqrt{a})), \quad F(x) \equiv x^{2} / a \quad(x \in[\sqrt{a}, a]),
$$

we have $d \mu(x)=a d x / x$.

(v) For $X \equiv \mathbf{R} \backslash(-1,1)$ and $F(x) \equiv 2 /(2-|x|)-1, d \mu(x)=\frac{d x}{2 x^{2}}$.

Example 3.5. Let $F$ be a map defined by the following graph:

Define $A \equiv\left(\begin{array}{lll}1 & 1 & 1 \\ 0 & 1 & 1 \\ 1 & 0 & 0\end{array}\right), \quad B \equiv \operatorname{diag}(1 / 3,1 / 2,1), R_{1} \equiv[0,1 / 3], R_{2} \equiv$

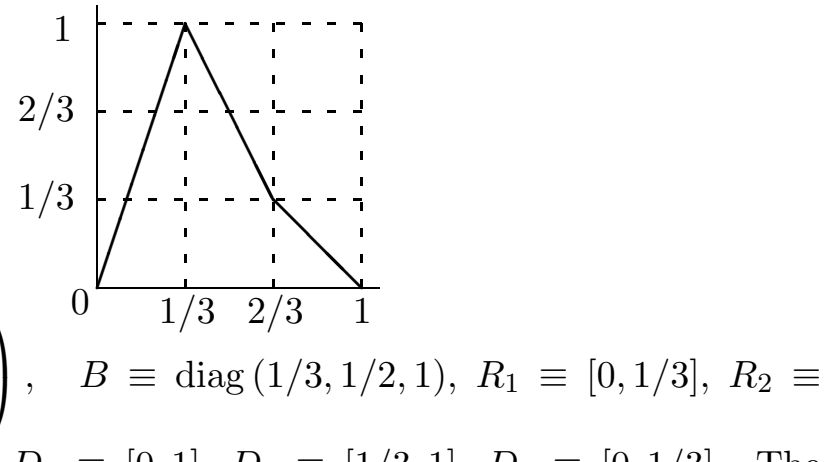
$[1 / 3,2 / 3], R_{3} \equiv[2 / 3,1], D_{1} \equiv[0,1], D_{2} \equiv[1 / 3,1], D_{3} \equiv[0,1 / 3]$. The $A$-branching function system $f=\left\{f_{1}, f_{2}, f_{3}\right\}, f_{i}: D_{i} \rightarrow R_{i}, i=1,2,3$, with the coding map $F$ is given by $f_{1}(x)=x / 3, \quad f_{2}(x)=-(x-1) / 2+$ $1 / 3, \quad f_{3}(x)=-x+1$. ¿From this and Theorem 1.2 (ii), $\left.P_{F}\right|_{W}=B A$ where $W \equiv \operatorname{Lin}<\left\{\chi_{R_{1}}, \chi_{R_{2}}, \chi_{R_{3}}\right\}>$. We see that $0,-1 / 6,1$ are eigenvalues of $\left.P_{F}\right|_{W}$. Hence they are eigenvalues of $P_{F}$.

Example 3.6. For $A \in M_{N}(\{0,1\})$ and an $A$-branching function system $f=\left\{f_{i}\right\}_{i=1}^{N}$ on a measure space $(X, \mu), \mu(X)<\infty$, assume that $b_{i} \equiv \Phi_{f_{i}}$ is constant for each $i=1, \ldots, N$. Then $b_{i}=r_{i} /\left(\sum_{j=1}^{N} a_{i j} r_{j}\right)$ 
where $r_{i} \equiv \mu\left(R_{i}\right)$. When $A \equiv\left(\begin{array}{ccc}0 & 1 & 1 \\ 1 & 0 & 1 \\ 1 & 1 & 1\end{array}\right)$, we have $B=\left(b_{1}, b_{2}, b_{3}\right)=$ $\operatorname{diag}\left(\frac{r_{1}}{r_{2}+r_{3}}, \frac{r_{2}}{r_{1}+r_{3}}, \frac{r_{3}}{r_{1}+r_{2}+r_{3}}\right)$. For $0<a<b<1$, consider the case $F$ on $X=[0,1]$ which graph is given as follows:
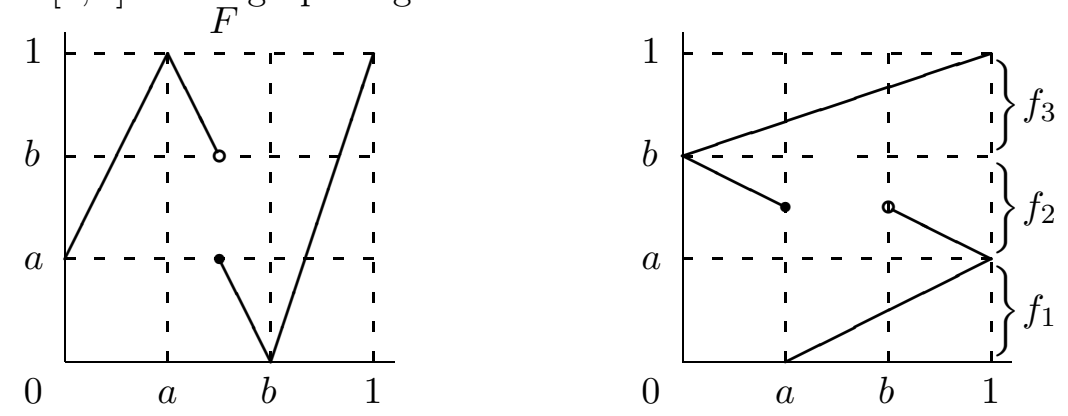

$F$ is the coding map of an $A$-branching function system given as follows: $f_{i}: D_{i} \rightarrow R_{i}, i=1,2,3$,

$$
\left\{\begin{array}{l}
f_{1}(x)=\frac{a}{1-a}(x-a) \quad\left(x \in D_{1}\right), \\
f_{2}(x)= \begin{cases}-\frac{b-a}{1-b+a} x+b, & \left(x \in R_{1}\right), \\
-\frac{b-a}{1-b+a}(x-1)+a & \left(x \in R_{3}\right),\end{cases} \\
f_{3}(x)=(1-b) x+b \quad(x \in[0,1]),
\end{array}\right.
$$

where $R_{1} \equiv[0, a], R_{2} \equiv[a, b], R_{3} \equiv[b, 1], D_{1} \equiv[a, 1], D_{2} \equiv[0, a] \cup(b, 1]$, $D_{3} \equiv[0,1]$. ¿From these, we have $B=\operatorname{diag}(a /(1-a),(b-a) /(1-b+a), 1-b)$.

Example 3.7. Let $F(x) \equiv x^{3}-3 x$ on $X \equiv[-2,2]$. Then $\frac{1}{\pi} \frac{d x}{\sqrt{4-x^{2}}}$ is a probabilistic invariant measure with respect to $F$. For a branching function system $f=\left\{f_{1}, f_{2}, f_{3}\right\}$ defined by $f_{1} \equiv\left(\left.F\right|_{[-2,-1]}\right)^{-1}, f_{2} \equiv\left(\left.F\right|_{[-1,1]}\right)^{-1}$, $f_{3} \equiv\left(\left.F\right|_{[1,2]}\right)^{-1},\left(L_{2}[-2,2], \pi_{f}\right)$ is $G P\left(3^{-1 / 2}, 3^{-1 / 2}, 3^{-1 / 2}\right)$ of $\mathcal{O}_{3}$.

Example 3.8. Let $X$ be the closed bounded region in $\mathbf{R}^{2}$ which is clipped by 4 -curves $L_{1} \equiv\left\{\left(-x^{2}, x\right): x \in[-1,1]\right\}, L_{2} \equiv\{(x,-1): x \in[-1,1]\}$, $L_{3} \equiv\left\{\left(2-x^{2}, x\right): x \in[-1,1]\right\}, L_{4} \equiv\{(x, 1): x \in[-1,1]\}$. Define a map $F$ on $X$ by

$$
F(x, y) \equiv\left(2 x^{2}+4 x y^{2}-2 y^{2}-4 x+1, \quad 2 y^{2}-1\right) \quad((x, y) \in X) .
$$

Then $F(X)=X$. There are the following four subregions $R_{1}, \ldots, R_{4}$ of $X$ : 

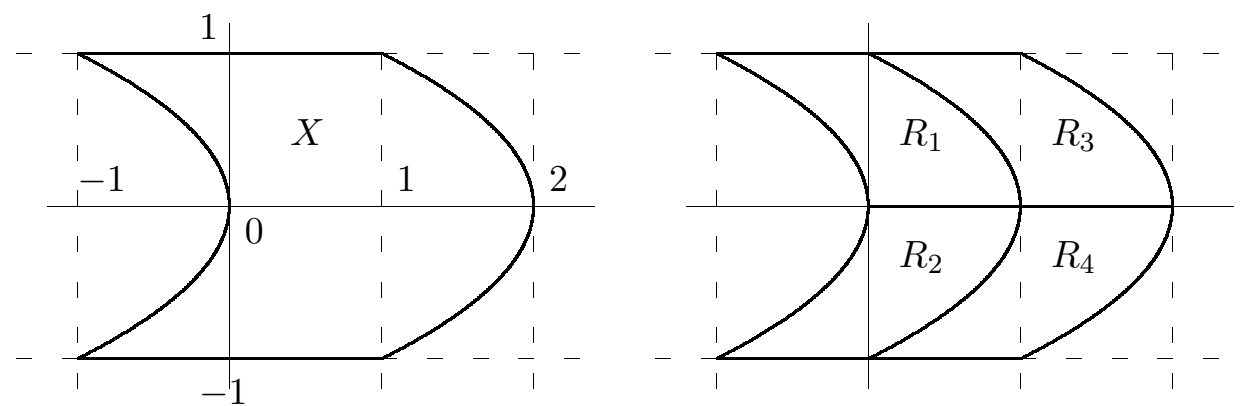

where the new center curve in the right figure is $\left\{\left(1-x^{2}, x\right): x \in[-1,1]\right\}$. Then $X=R_{1} \cup \cdots \cup R_{4}$ and $\left.F\right|_{R_{i}}$ is a bijection from $R_{i}$ to $X$ for each $i=1, \ldots, 4$. Then

$$
d \mu(x, y)=\frac{1}{\pi^{2}} \frac{d x d y}{\sqrt{\left(1-y^{2}\right)\left(x+y^{2}\right)\left(2-x-y^{2}\right)}}
$$

is a probabilistic invariant measure on $X$ with respect to $F$. For a branching function system $f=\left\{f_{i}\right\}_{i=1}^{4}$ defined by $f_{i} \equiv\left(\left.F\right|_{R_{i}}\right)^{-1},\left(L_{2}(X, d x d y), \pi_{f}\right)$ is $G P(1 / 4,1 / 4,1 / 4,1 / 4)$ of $\mathcal{O}_{4}$.

Acknowledgement: I would like to thank Makoto Mori for his talk in [7].

\section{References}

[1] O.Bratteli and P.E.T.Jorgensen, Iterated function Systems and Permutation Representations of the Cuntz algebra, Memories Amer. Math. Soc. No.663 (1999).

[2] J.Cuntz, Simple $C^{*}$-algebras generated by isometries, Comm. Math. Phys. 57 (1977), 173-185.

[3] J.Cuntz and W.Krieger, A class of $C^{*}$-algebra and topological Markov chains, Invent.Math., 56 (1980), 251-268.

[4] A.Lasota and J.A.Yorke, Exact dynamical systems and the Frobenius-Perron operator, Trans.Amer.Math.Soc. 273, (1982), 375-384.

[5] A.Lasota and M.C.Mackey, Chaos, Fractals and Noise, Stochastic Aspects of Dynamics, Second Edition, Springer-Verlag (1991).

[6] M.Mori, On the convergence of the spectrum of Perron-Frobenius operators, Tokyo J. Math. 17 (1994), 1-19.

[7] M.Mori, Fractal and Perron-Frobenius operator (Japanese), Representations of Cuntz algebras and their applications in mathematical physics, Symposium in RIMS Kōkyuroku No.1333 (2003).

[8] S.M.Ulam and J.von Neumann, On combination of stochastic and deterministic processes, Bull. Am. Math. Soc., 53:1120, (1947). 